Editorial

\title{
Green revolution towards nanobiotechnology
}

Volume 2 Issue I - 2015

\section{Editorial}

The field of "Nanobiotechnology" is growing day by day with respect to people with needs in concern of drug delivery, cosmetics and environmental applications. The surface to volume ratio of these nanosized particles is high then compared to micro and macro sized particles. Hence they are easily attracted with the biological environment and delivered the target particles to target site. The RNCOS (News Letter) reported the global market for nano based materials will be reached around $30 \%$ (US\$ 1.6 trillion) in 20092013 but uses of nanoparticles in biomedical applications are reached around 51\% during 2011-2014. From the $20^{\text {th }}$ century, the biosynthesis of nanoparticles of environmentally benign materials like plant, microalgae, bacteria, fungi and animals has been increased. Green synthesis provides advancement over chemical and physical method as it is cost effective, environment friendly, easily scaled up for large scale synthesis and in this method there is no need to use high pressure, energy, temperature and toxic chemicals. Unsurprisingly, the phenolic compounds are abundant in the plant kingdom are of particular interest because of their potential biological properties, such as an antioxidant, chelating, free radical scavenging, anti-inflammatory, anti-allergic, anti-microbial, anti-viral, and anti-carcinogenic. It is important to optimize the various process variables influenced in the bio-reduction process green nanoparticles.

Due to thermal kinetics, larger surface of nanoparticles it facilitates the aggregation or friction, which may affect their efficiency in drug development. The aggregated nanoparticles are accumulated in organs and cause toxicity mediated failure to organ functions under clinical trials. Recent reports revealed that tissue distribution of gold nanoparticles is size dependent with the smallest nanoparticles (15$50 \mathrm{~nm}$ ) showing the most widespread organ distribution including liver, lung, spleen, kidney, brain, heart and stomach (gastrointestinal absorption) of experimental mice. Than compared to crude extract mediated biosynthesis researchers focussed their keen observation

\author{
Satyavani K, Gurudeeban S \\ Department of Molecular Biology, Istanbul Medeniyet University, \\ Turkey
}

Correspondence: Gurudeeban S, Department of Molecular Biology, Faculty of Science, Istanbul Medeniyet University, Goztepe 34730, Istanbul, Turkey, Tel 905061555476

Email gurudeeb99@gmail.com

Received: November 27, 2014 | Published: December 23, 2014

towards isolation of specific secondary metabolites and its role on nanoparticles synthesis. İt provides better stability and capping on the synthesized nanoparticles. However the selection of suitable novel nanocarrier system like Solid lipid nanoparticles, PLGA is increased to deliver the drug molecules into biological system for treatment of metabolic disorders (eg. Type 2 Diabetes Mellitus) with non toxic, increased bioavailability and stability.

This emerging plant mediated nanoparticle synthesis might be preventing the various disorders related to human health. Before that complete knowledge about the exact mechanism of green nanoparticles with biological environment will be clearly known by the researchers to find out effective non toxic nanodrug. Also more comprehensive studies will be needed regarding this toxicity towards the environment.

\section{Acknowledgments}

None.

\section{Conflicts of interest}

None. 